\title{
Анализ особенностей деградации, вызываемой горячими носителями, в транзисторах с каналом в форме плавника
}

\author{
(ㄷ А.А. Макаров ${ }^{1}$, С.Э. Тягинов ${ }^{1,2}$, B. Kaczer ${ }^{3}$, M. Jech ${ }^{1}$, A. Chasin ${ }^{3}$, \\ A. Grill ${ }^{1}$, G. Hellings ${ }^{3}$, М.И. Векслер ${ }^{2, \uparrow, ~ D . ~ L i n t e n ~}{ }^{3}$, T. Grasser $^{1}$ \\ ${ }^{1}$ TU Vienna, Institute for Microelectronics, \\ Vienna 1040, Austria \\ ${ }^{2}$ Физико-технический институт им. А.Ф. Иоффре Российской академии наук, \\ 194021 Санкт-Петербург, Россия \\ ${ }^{3}$ IMEC, Kapeldreef 75, \\ 3001 Leuven, Belgium \\ ฯ E-mail: vexler@mail.ioffe.ru
}

(Получена 24 февраля 2018 г. Принята к печати 27 февраля 2018 г.)

\begin{abstract}
Впервые проведено моделирование деградации, вызываемой горячими носителями (ДВГН), в непланарных полевых транзисторах с каналом в форме плавника (FinFET). Для этого использована физическая модель, которая рассматривает одночастичные и многочастичные процессы разрыва связей кремний-водород, а также их суперпозиции. Для вычисления темпа диссоциации связей используются функции распределения носителей по энергии, которые находят при решении транспортного уравнения Больцмана. С помощью анализа ДВГН показано, что деградация локализована в части канала, примыкающей к стоку транзистора, в районе верхней стенки канала. Хорошее соответствие между экспериментальными и расчетными деградационными характеристиками было получено с теми же параметрами модели, которые применялись при воспроизведении ДВГН в планарных короткоканальных транзисторах, а также в мощных полупроводниковых приборах.
\end{abstract}

DOI: $10.21883 /$ FTP.2018.10.46457.8820

\section{1. Введение}

Миниатюризация ключевого элемента современной микроэлектроники - полевого транзистора (ПТ; английский термин field-effect transistor, FET) - подразумевает не только уменьшение линейных размеров прибора и толщины подзатворного диэлектрика, но и оптимизацию потребляемой мощности, а также обеспечение надлежащего электростатического управления каналом [1]. Требующими решения физическими задачами при этом являются увеличение крутизны подпороговых характеристик приборов (subthreshold slope) и снижение тока выключенного состояния (OFF current). На момент появления указанных требований потенциал оптимизации транзисторов „традиционной“ планарной архитектуры был исчерпан, что привело к появлению приборов новых, трехмерных, топологий - мультизатворных структур (multi-gate FET, MGFET), транзисторов с каналом в форме плавника (FinFET), а также нанопроволочных ПТ (nanowire FET, NWFET) [2,3]. Наиболее перспективным из указанных приборов является транзистор с каналом в форме плавника $[4,5]$.

Важнейшим критерием „жизнеспособности“ каждого технологического поколения, наряду с производительностью и потребляемой мощностью, является надежность полупроводниковых приборов. Обеспечение надежности ПТ предполагает борьбу с целым рядом паразитных эффектов. Применительно к транзисторам с каналом в форме плавника недавно было показано, что основным механизмом разрушения диэлектрического слоя их затворной секции является деградация, вызываемая горячими носителями (ДВГН, английский термин hot carrier degradation, HCD) [5].

В последние несколько лет эффект ДВГН в ПТ с каналом в форме плавника был объектом интенсивных экспериментальных [6-8] и теоретических $[9,10]$ исследований, но природа данного эффекта до конца так и не понята, а надежная предиктивная модель, основанная на физических принципах, пока не предложена. Попытки моделирования ДВГН в приборах этого класса были основаны на эмпирических и феноменологических упрощениях. Они сводились к оценке времени жизни прибора в реальном режиме стрессового воздействия горячими носителями посредством экстраполяции данных, полученных при более высоких напряжениях стресса. Однако, как показывает практика [11-13], переход от одного режима к другому влечет смену основного микроскопического механизма, ответственного за ДВГН, что делает указанную экстраполяцию времени жизни прибора несостоятельной.

Немногочисленные модели, в рамках которых предлагалось описание физических механизмов, ответственных за ДВГН, использовали темп ударной ионизации как количественный критерий интенсивности разрушения транзистора под воздействием горячих носителей (см., например, [9]). Такой подход представляется спорным, потому что генерация дефектов в ходе ДВГН связана с разрывом связей кремний-водород $(\mathrm{Si}-\mathrm{H})$ на границе раздела диэлектрик/кремний и темп данного процесса имеет совершенно другую зависимость от энергии час- 
тиц, чем скорость ударной ионизации. Более того, в рамках подобных подходов полагается, что темп ударной ионизации является функцией электрического поля; при этом ранее нами было показано, что темп разрыва связей $\mathrm{Si}-\mathrm{H}$ (и, как следствие, концентрация интерфейсных состояний $\left.N_{i t}\right)$ следует изменениям электрического поля со значительной задержкой $[14,15]$. Наконец, в современных транзисторах рабочее напряжение приборов нередко оказывается ниже $1 \mathrm{~B}$, т.е. темп ударной ионизации пренебрежимо мал и не может быть использован для моделирования ДВГН.

Для описания ДВГН в транзисторах с каналом в форме плавника в данной работе мы применяем физическую модель, основанную на моделировании транспорта носителей в полупроводниковых структурах.

\section{2. Модель деградации, вызываемой горячими носителями}

Для моделирования и анализа особенностей ДВГН мы используем методологию, разработанную нами ранее [16-19] и применявшуюся для описания повреждения приборов горячими носителями в короткоканальных транзисторах и в мощных полупроводниковых приборах, т.е. в транзисторах, изготовленных методом двойной диффузии (lateral double-diffused MOS transistor, LDMOS) [20,21]. Данная модель может быть условно разделена на три основных блока: моделирование транспорта носителей в полупроводниковой структуре; описание микроскопических механизмов генерации дефектов на границе раздела диоксид кремния/кремний $\left(\mathrm{SiO}_{2} / \mathrm{Si}\right)$; моделирование характеристик поврежденных приборов.

Для моделирования транспорта носителей нами применяется симулятор ViennaSHE, осуществляющий детерминистическое решение (в отличие от стохастических подходов, основанных на методе МонтеКарло) транспортного уравнения Больцмана (Boltzmann transport equation) [22,23]. Вычисления проводятся с учетом реальной зонной структуры $\mathrm{Si}$, а также различных механизмов рассеяния, а именно: рассеяния на ионизованной примеси, рассеяния на границе раздела, ударной ионизации, а также электрон-фононных и электрон-электронных взаимодействий.

C помощью программы ViennaSHE находятся функции распределения (ФР; английский термин distribution function, DF) носителей по энергии для заданной apхитектуры прибора и условий стрессового воздействия. ФР затем используются для вычисления темпов разрыва связей $\mathrm{Si}-\mathrm{H}$. В рамках модели рассматриваются два механизма разрыва - одночастичный и многочастичный процессы. Первый механизм соответствует классической ДВГН, когда пакет носителей содержит значительное количество горячих частиц с энергиями $E>E_{a}$ (где $E_{a}=2.6$ эВ - энергия разрыва связи $\mathrm{Si}-\mathrm{H}[24]$ ). Второй механизм типичен для низких напряжений стрессового

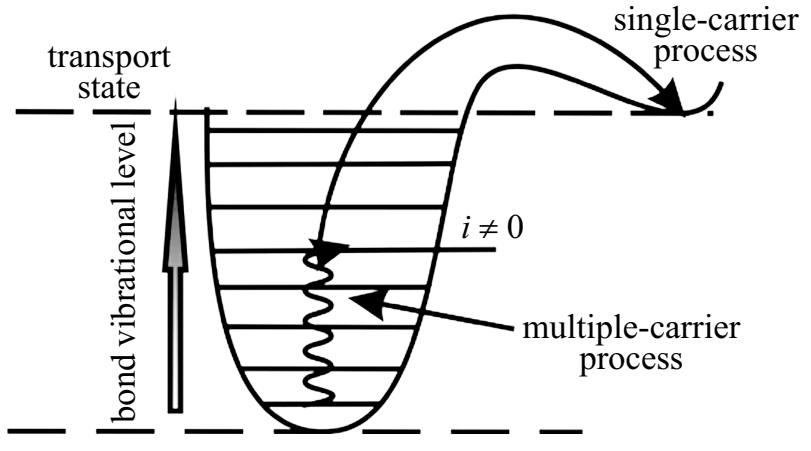

Рис. 1. Схема одночастичного и многочастичного механизмов диссоциации связи $\mathrm{Si}-\mathrm{H}$.

воздействия, когда диссоциация связи провоцируется каскадным воздействием холодных носителей, индицирующих многочастичное возбуждение колебательных мод связи [11,17,18,25-27]. Наша модель учитывает все суперпозиции этих двух механизмов. Нами было показано [18,21,23], что наиболее эффективным процессом деградации связи является сценарий, согласно которому она сначала возбуждается серией холодных частиц до некоторого промежуточного связанного состояния (с требуемой энергий разрыва менее номинальной $E_{a}$ ), а потом разрывается горячим носителем (с $\left.E<E_{a}\right)-$ см. рис. 1.

Напомним, что темп диссоциации связи по одночастичному механизму записывается как $[17,28]$

$$
I\left(E_{a}\right)=\int f(E) \rho(E) \sigma\left(E, E_{a}\right) v(E) d E+I_{\mathrm{th}},
$$

где $f(E) \rho(E)$ - обобщенная ФР (произведение числа заполнения $f$ на плотность состояний $\rho$, имеет размерность эВ $\left.{ }^{-1} \cdot \mathrm{cm}^{-3}\right), \sigma\left(E, E_{a}\right)$ - энергозависимое сечение реакции разрыва связи с соответствующей энергией активации $E_{a}$, а $v(E)$ - групповая скорость частиц; интегрирование в (1) осушествляется по всем энергиям частиц в ансамбле. Член $I_{\text {th }}$ описывает термическую активацию атома Н через барьер, разделяющий связанное и транспортное состояния.

При учете многочастичного возбуждения связи на уровень $i$ (с энергией $E_{i}$ ) потенциальный барьер между этим уровнем и транспортной модой понижается на величину $E_{i}$, а соответствующий темп вычисляется как

$$
\begin{aligned}
I_{i}\left(E_{a}\right)= & \int f(E) \rho(E) \sigma\left(E-E_{i}\right) v(E) d E \\
& +\omega_{\text {th }} \exp \left(-\frac{E-E_{i}}{k T}\right)
\end{aligned}
$$

где $\omega_{\text {th }}$ - „частота попыток“ термического разрыва, $k$ - постоянная Больцмана, $T-$ решеточная температура. Далее кумулятивный темп разрыва связей находится в виде суммы вкладов отдельных уровней, т.е. $I\left(E_{a}\right)=\Sigma I_{i}\left(E_{a}\right)$. 
Как для одночастичного, так и для многочастичного механизмов сечения рассеяния моделируются выражением Келдыша:

$$
\sigma^{\mathrm{SP} \mid \mathrm{MP}}\left(E, E_{a}\right)=\sigma_{0}^{\mathrm{SP} \mid \mathrm{MP}}\left(\frac{E-E_{\mathrm{th}}}{1 \mathrm{eV}}\right)^{p^{\mathrm{SP} \mid \mathrm{MP}}}
$$

с различными значениями параметров $\sigma_{0}^{\mathrm{SP} \mid \mathrm{MP}}$ и $p^{\mathrm{SP} \mid \mathrm{MP}}$ $\left(p^{\mathrm{SP}}=12\right.$ и $\left.p^{\mathrm{MP}}=1\right)$; индексом „SP“ обозначен одночастичный процесс, а „МР“ - многочастичный. Для одночастичного процесса $E_{\mathrm{th}}=E_{a}$, а для многочастичного $E_{\mathrm{th}}$ соответствует расстоянию между уровнями осциллятора (см. рис. 1).

В рамках модели мы полагаем [18], что диссоциация связи протекает через моду растяжения (stretching mode); вообще говоря, связь имеет вторую колебательную моду, моду закручивания (bending mode), которая, однако, не связана с ее разрывом [24]. Также мы полагаем, что энергия активации является статистически флуктуирующей величиной, описываемой нормальным распределением со средним значением $E_{a}=2.6$ эВ [24] и среднеквадратичным отклонением $\sigma_{E}=0.22$ эВ. Значение $\sigma_{E}$ является одним из двух подгоночных параметров модели наряду с концентрацией пассивных связей $\mathrm{Si}-\mathrm{H}$ на интерфейсе $\left(N_{0}=5.6 \cdot 10^{12} \mathrm{~cm}^{-2}\right)$. Обе эти величины зависят от конкретного технологического процесса, но идентичны для транзисторов, изготовленных при одинаковых условиях. Значения других параметров фиксированы: например, мы используем $\sigma_{0}^{\mathrm{SP}}=5 \cdot 10^{-18} \mathrm{~cm}^{2}$ и $\sigma_{0}^{\mathrm{MP}}=5 \cdot 10^{-19} \mathrm{~cm}^{2}$, т. е. значения, идентичные тем, что применялись для моделирования ДВГН в планарных транзисторах [18].

Рассчитанные величины темпов генерации дефектов далее применяются для вычисления координатно-зависимой плотности интерфейсных состояний $N_{i t}$ для каждого значения времени стресса. Полученные плотности потом используются в программе-симуляторе MiniMOS-NT [29], которая моделирует изменение характеристик прибора со временем. При этом учитываются как локальные электростатические возмущения, связанные с наличием заряженных ловушек, так и снижение подвижности носителей вследствие появления дополнительных рассеивающих центров.

\section{3. Приборы и эксперимент}

Для исследования деградации приборов при воздействии горячих носителей мы использовали транзисторы с каналом в форме плавника, которые имеют трапециевидное сечение (см. рис. 2). Длина электрода затвора этих приборов равна $40 \mathrm{Hм}$, а длина канала $28 \mathrm{нм}$; рабочее и пороговое напряжения составляли $V_{D D}=0.9 \mathrm{~B}$ и $V_{\text {th }} \approx 0.4 \mathrm{~B}$ соответственно. Слой подзатворного диэлектрика составной и включает две компоненты: пленки диоксида кремния $\left(\mathrm{SiO}_{2}\right)$ и диоксида гафния $\left(\mathrm{HfO}_{2}\right)$. Эквивалентная электрическая толщина (effective oxide thickness, EOT) результирующего слоя равнялась $1.2 \mathrm{Hм}$.

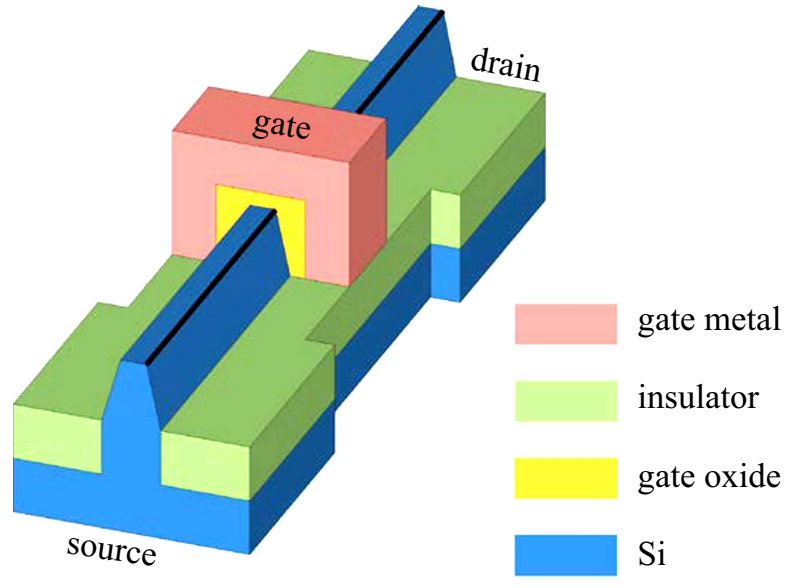

Рис. 2. Схематическое изображение изучаемого транзистора с каналом в форме плавника. Сечение канала имеет форму трапеции.

Приборы были изготовлены по стандартному технологическому процессу на IMEC (см. [30]).

Транзисторы были подвергнуты стрессовому воздействию горячими носителями при напряжениях, соответствующих наиболее сильной ДВГН, т.е. при $V_{g s} \approx V_{d s}$ (где $V_{d s}, V_{g s}-$ напряжения сток-исток и затвор-исток) [12,13,31], и комнатной температуре. Отметим, что мы независимо выбирали значения $V_{d s}$, a $V_{g s}$ подгоняли таким образом, чтобы обеспечить $V_{g s}-V_{\text {th }}=V_{d s}$. В итоге использовались следующие комбинации смещений: $V_{d s}=1.6 \mathrm{~B}, V_{g s}=1.7 \mathrm{~B} ; V_{d s}=1.7 \mathrm{~B}$, $V_{g s}=1.8 \mathrm{~B} ; V_{d s}=1.8 \mathrm{~B}, V_{g s}=1.9 \mathrm{~B}$. В качестве количественной характеристики (метрики) ДВГН использовалось относительное изменение линейного тока стока $\Delta I_{d, \operatorname{lin}}(t)=\left[I_{d, \operatorname{lin}}(t)-I_{d, \operatorname{lin} 0}\right] / I_{d, \operatorname{lin} 0}$, где $t-$ время деградации, а $I_{d, \operatorname{lin} 0}$ - ток стока неповрежденного ПТ, который соответствует $V_{d s}=0.05 \mathrm{~B}$ и $V_{g s}=0.9 \mathrm{~B}$.

Предварительно был исследован вопрос, имеем ли мы дело исключительно с деградацией вследствие генерации интерфейсных состояний или же следует также учитывать вклад, связанный с зарядкой/перезарядкой ловушек в толще диэлектрического слоя. Последняя компонента является восстанавливаемой [32] и была изучена в работе [30] для широкого диапазона напряжений стрессового воздействия, причем использовались транзисторы того же технологического процесса, что и наши образцы. Оказалось, что восстановления после деградации не происходит в течение достаточно длинного промежутка времени, и, следовательно можно утверждать, что ДВГН в наших образцах заключается только в генерации дефектов путем разрыва связей $\mathrm{Si}-\mathrm{H}$.

\section{4. Результаты и обсуждение}

На рис. 3 показано семейство обобщенных функций распределения электронов по энергии, вычисленных 
для двух комбинаций напряжений стрессового воздействия, т.е. для $V_{d s}=1.6 \mathrm{~B}, V_{g s}=1.7 \mathrm{~B}$ и $V_{d s}=1.8 \mathrm{~B}$, $V_{g s}=1.9 \mathrm{~B}$. ФР построены для ребра между верхней границей канала и его боковой стенкой (жирная линия на рис. 2), для трех латеральных позиций (направление сток-исток, обозначенное осью $x$, см. рис. 2 ): в области истока, в центре канала и в области стока (рис. 2). Видно, что в районе истока термализованные электроны описываются распределением Максвелла, а в центре канала и в районе стока эти функции сильно неравновесные.

Профили плотности состояний ловушек, вычисленные для $V_{d s}=1.7 \mathrm{~B}, V_{g s}=1.8 \mathrm{~B}$ и времени стрессового воздействия $t \approx 200 \mathrm{c}$, показаны на рис. 4. Данные профили $N_{i t}(x, y, z)$ позволяют констатировать, что деградация локализована в верхней части стороны канала, граничащей со стоком. Кроме того, видно, что для любой латеральной позиции $x$ величина $N_{i t}$ увеличивается в сторону вершины канала (в направлении увеличения координаты $y$ ). Такое поведение связано с наличием заметной вертикальной компоненты поля и разогревом носителей не только в направлении исток-сток, но и вдоль оси $y$. На рис. 5 показана эволюция плотности $N_{i t}$ со временем для более мягких условий стрессового воздействия $V_{d s}=1.6 \mathrm{~B}, V_{g s}=1.7 \mathrm{~B}$ (как и ФР на рис. 3, профили $N_{i t}$ построены для ребра между вершиной и боковой стенкой канала). Видно, что наиболее сильно повреждается область стока, что проявляется, например, в насыщении ДВГН при длительных стрессах $(t=200$ с и 2 кс), а временна́я зависимость ДВГН определяется распространением фронта $N_{i t}$ в сторону стока.

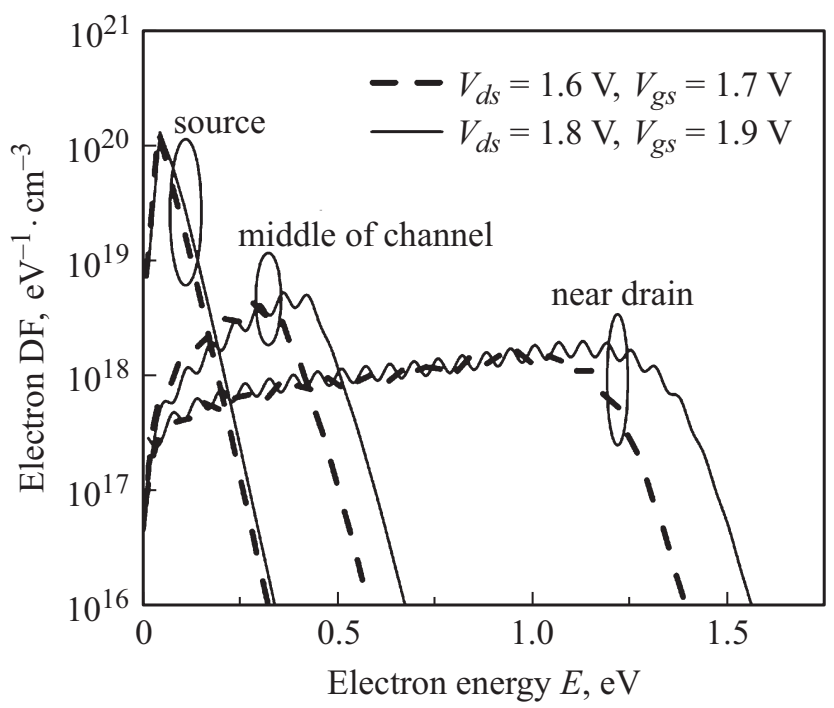

Pис. 3. Функция распределения (DF) электронов по энергии, вычисленные с помощью программы-симулятора ViennaSHE для $V_{d s}=1.6 \mathrm{~B}, V_{g s}=1.7 \mathrm{~B}$ и $V_{d s}=1.8 \mathrm{~B}, V_{g s}=1.9 \mathrm{~B}$. ФР выведены для ребра между верхней плоскостью канала и его боковой стенкой в области истока, в центре прибора и в районе стока.

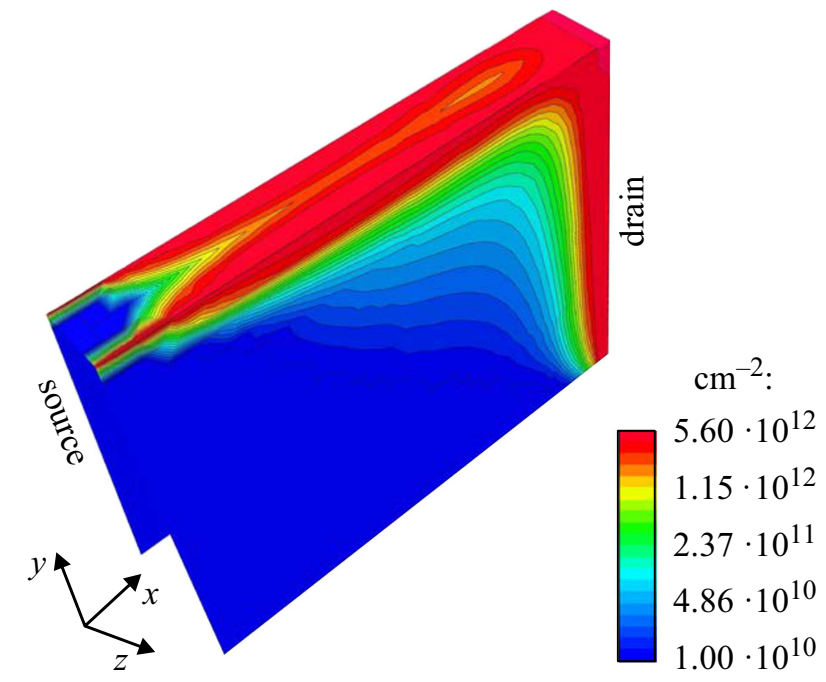

Рис. 4. Профили плотности ловушечных состояний на границе раздела канал/диэлектрик, $N_{i t}(x, y, z)$, для $V_{d s}=1.7 \mathrm{~B}$, $V_{g s}=1.8 \mathrm{~B}$ и $t \approx 200 \mathrm{c}$. Видно, что наиболее поврежденное место в приборе соответствует верхней части стоковой стороны канала.

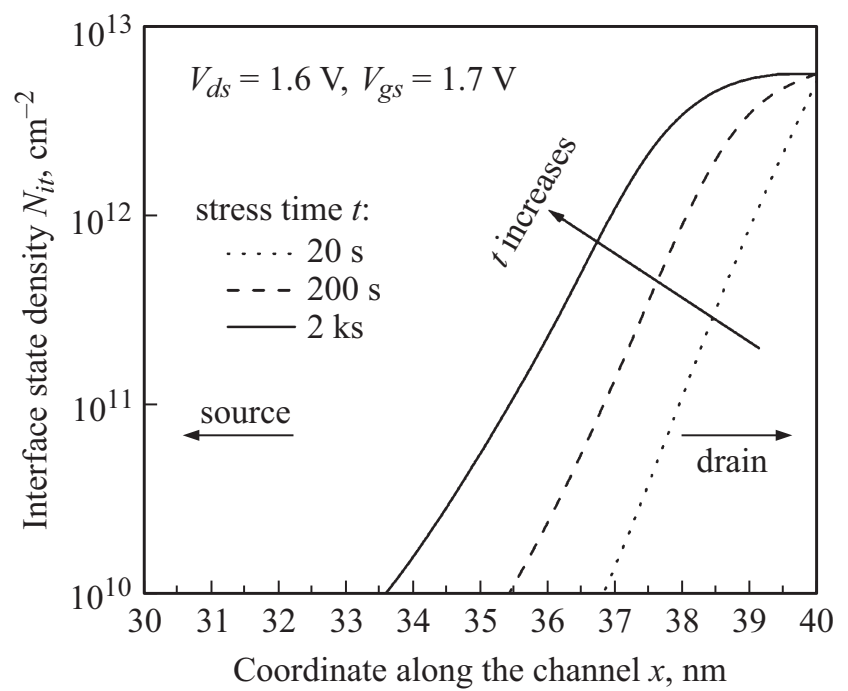

Рис. 5. Эволюция профилей $N_{i t}(x)$ со временем для $V_{d s}=1.6 \mathrm{~B}, V_{g s}=1.7 \mathrm{~B}$. Представлены данные для ребра между верхней плоскостью канала и его боковой стенкой.

Наконец, из рис. 6 следует, что модель способна воспроизвести экспериментальные зависимости $\Delta I_{d \text {,lin }}(t)$ c хорошей точностью для всех комбинаций стрессовых напряжений. Весьма важным представляется тот факт, что значения параметров, использованные для моделирования изменения характеристик транзисторов с формой канала в виде плавника, практически идентичны тем, которые были задействованы для описания ДВГН в планарных транзисторах с длиной канала 65 нм, а также в мощных транзисторах, изготовленных методом двойной диффузии с длиной канала $\sim 1.0$ мкм. Это свидетельствует об универсальности нашей модели и 


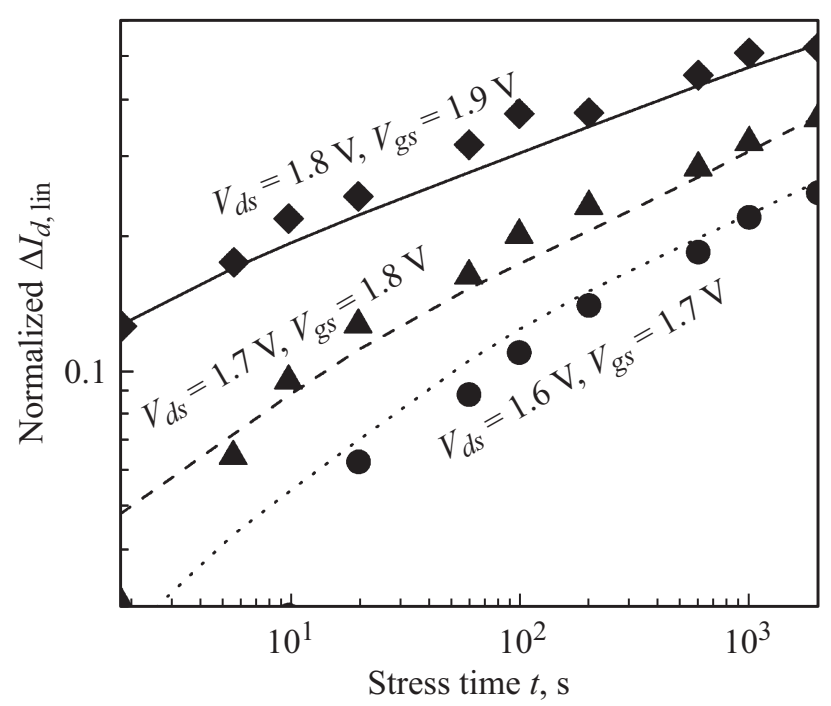

Рис. 6. Сравнение экспериментальных и расчетных характеристик $\Delta I_{d, \operatorname{lin}}(t)$.

позволяет утверждать, что с ее помощью можно прогнозировать время жизни различных приборов при рабочих напряжениях.

\section{5. Заключение}

В данной работе впервые была применена физическая модель деградации, вызываемой горячими носителями, для анализа особенностей этого паразитного эффекта в полевых транзисторах с каналом в форме плавника (FinFET). Модель основана на анализе транспорта носителей в полупроводниковых структурах и рассматривает взаимодействие одночастичного и многочастичного механизмов разрыва связей кремний-водород. Благодаря этому модель применима в широком диапазоне изменения параметров ПТ, т.е. хорошо описывает ДВГН как в режиме стресса с достаточно высокими напряжениями сток-исток и затвор-исток, так и в более „щадящих“ режимах, близких к рабочим.

Впервые выявлено, где именно локализовано повреждение, вызванное горячими носителями, - в верхней части канала, примыкающей к стоку. Другой обнаруженной особенностью ДВГН в транзисторах с каналом в форме плавника является увеличение концентрации дефектов по мере приближения к верхней грани канала. Это связано с вертикальной составляющей электрического поля, которое разгоняет носители не только в направлении исток-сток, но и в направлении от основания канала к его верхней грани.

Важно отметить, что набор параметров, использованный в данной работе, практически идентичен значениям, ранее задействованным для моделирования ДВГН в планарных короткоканальных транзисторах, а также в мощных полупроводниковых приборах. Это обстоятель- ство позволяет утверждать, что наша модель является универсальной и предикативной.

\section{Список литературы}

[1] Interational Technology Roadmap for Semiconductors (ITRS), Chap. 5: More Moore (2015).

[2] F. Isabelle, C.A. Colinge, J.-P. Colinge. Nature, 479 (7373), 310 (2011).

[3] J.-P. Colinge, C.-W. Lee, A. Afzalian, N.D. Akhavan, R. Yan, I. Ferain, P. Razavi, B. O’Neill, A. Blake, M. White, A.-M. Kelleher, B. McCarthy, R. Murphy. Nature Nano, 5 (3), 225 (2010).

[4] C. Auth, C. Allen, A. Blattner, D. Bergstrom, M. Brazier, M. Bost, M. Buehler, V. Chikarmane, T. Ghani, T. Glassman, R. Grover, W. Han, D. Hanken, M. Hattendorf, P. Hentges, R. Heussner, J. Hicks, D. Ingerly, P. Jain, S. Jaloviar, R. James, D. Jones, J. Jopling, S. Joshi, C. Kenyon, H. Liu, R. McFadden, B. McIntyre, J. Neirynck, C. Parker, L. Pipes, I. Post, S. Pradhan, M. Prince, S. Ramey, T. Reynolds, J. Roesler, J. Sandford, J. Seiple, P. Smith, C. Thomas, D. Towner, T. Troeger, C. Weber, P. Yashar, K. Zawadzki, K. Mistry. Proc. Symp. on VLSI Technology (VLSIT) (2012) p. 131.

[5] S. Novak, C. Parker, D. Becher, M. Liu, M. Agostinelli, M. Chahal, P. Packan, P. Nayak, S. Ramey, S. Natarajan. Proc. IEEE Intern. Reliability Phys. Symp. (2015) Paper no. 2F.2.

[6] D.H. Lee, S.M. Lee, C.G. Yu, J.T. Park. IEEE Electron Dev. Lett., 32 (9), 1176 (2011).

[7] S. Ramey, A. Ashutosh, C. Auth, J. Clifford, M. Hattendorf, J. Hicks, R. James, A. Rahman, V. Sharma, A. St. Amour, C. Wiegand. Proc. IEEE Intern. Reliability Phys. Symp. (IRPS) (2013) Paper no. 4C.5.

[8] M. Cho, P. Roussel, B. Kaczer, R. Degraeve, J. Franco, M. Aoulaiche, T. Chiarella, T. Kauerauf, N. Horiguchi, G. Groeseneken. IEEE Trans. Electron Dev., 60 (12), 4002 (2013).

[9] C.-D. Young, J.-W. Yang, K. Matthews, S. Suthram, M.M. Hussain, G. Bersuker, C. Smith, R. Harris, R. Choi, B.H. Lee, H.-H. Tseng. J. Vac. Sci. Technol. B, 27 (1), 468 (2009).

[10] I. Messaris, T.A. Karatsori, N. Fasarakis, C.G. Theodorou, S. Nikolaidis, G. Ghibaudo, C.A. Dimitriadis. Microelectron. Reliab., 56, 10 (2016).

[11] A. Bravaix, C. Guerin, V. Huard, D. Roy, J. Roux, E. Vincent. Proc. Intern. Reliability Phys. Symp. (IRPS) (2009) p. 531.

[12] S. Rauch, G. La Rosa. Proc. Intern. Reliability Phys. Symp. (IRPS) (2010) tutorial.

[13] S. Tyaginov, T. Grasser. Proc. Intern. Integr. Reliability Workshop (IIRW) (2012) p. 206.

[14] I. Starkov, S. Tyaginov, H. Enichlmair, J. Cervenka, C. Jungemann, S. Carniello, J.M. Park, H. Ceric, T. Grasser. J. Vac. Sci. Technol. B, 29 (1), Paper no. 01AB09 (2011).

[15] S. Tyaginov, I. Starkov, C. Jungemann, H. Enichlmair, J.M. Park, T. Grasser. Proc. European Solid-State Device Research Conf. (ESSDERC) (2011) p. 151.

[16] S.E. Tyaginov, I.A. Starkov, O. Triebl, J. Cervenka, C. Jungemann, S. Carniello, J.M. Park, H. Enichlmair, M. Karner, Ch. Kernstock, E. Seebacher, R. Minixhofer, H. Ceric, T. Grasser. Microelectron. Reliab., 50, 1267 (2010).

[17] M. Bina, S. Tyaginov, J. Franco, K. Rupp, Y. Wimmer, D. Osintsev, B. Kaczer, T. Grasser. IEEE Trans. Electron Dev., 61 (9), 3103 (2014). 
[18] S. Tyaginov, M. Jech, J. Franco, P. Sharma, B. Kaczer, T. Grasser. IEEE Electron Dev. Lett., 37 (1), 84 (2016).

[19] С.Э. Тягинов, А.А. Макаров, М. Јесh, М.И. Векслер, J. Franco, B. Kaczer, T. Grasser. ФTT, 5 (2), 254 (2018).

[20] P. Sharma, S. Tyaginov, Y. Wimmer, F. Rudolf, K. Rupp, M. Bina, H. Enichlmair, J.-M. Park, R. Minixhofer, H. Ceric, T. Grasser. IEEE Trans. Electron Dev., 62 (6), 1811 (2015).

[21] P. Sharma, S. Tyaginov, M. Jech, Y. Wimmer, F. Rudolf, H. Enichlmair, J.-M. Park, H. Ceric, T. Grasser. Solid-State Electron., 115, Part B, 185 (2016).

[22] K. Rupp, T. Grasser, A. Jungel. Proc. Intern. Electron Devices Meeting (IEDM) (2011) p. 789.

[23] M. Bina, K. Rupp, S. Tyaginov, O. Triebl, T. Grasser. Proc. Intern. Electron Devices Meeting (IEDM) (2012) p. 713.

[24] K.L. Brower. Phys. Rev. B, 42 (6), 3444 (1990).

[25] K. Hess, I.C. Kizilyalli, J.W. Lyding. IEEE Trans Electron Dev., 45 (2), 406 (1998)

[26] W. McMahon, K. Hess. J. Comput. Electron., 1 (3), 395 (2002).

[27] C. Guerin, V. Huard, A. Bravaix. J. Appl. Phys., 105, 114513 (2009).

[28] S. Tyaginov, M. Bina, J. Franco, D. Osintsev, O. Triebl, B. Kaczer, T. Grasser.Proc. Intern. Reliability Phys. Symp. (IRPS), (2014) Paper no. XT.16.

[29] MiniMOS-NT Device and Circuit Simulator (Institute for Microelectronic, TU Wien).

[30] A. Chasin, J. Franco, R. Ritzenthaler, G. Hellings, M. Cho, Y. Sasaki, A. Subirats, P. Roussel, B. Kaczer, D. Linten, N. Horiguchi, G. Groeseneken, A. Thean. Proc. IEEE Intern. Reliability Phys. Symp. (IRPS) (2016) Paper no. 4B-4.

[31] A. Bravaix, V. Huard. Proc. Eur. Symp. Reliability of Electron Devices Failure Physics and Analysis (ESREF) (2010) p. 1267.

[32] T. Grasser. Microelectron. Reliab., 52 (1), 39 (2012).

Редактор Л.В. Шаронова

\section{Analysis of hot-carrier degradation features in FinFETs}

A.A. Makarov ${ }^{\mathbf{1}}$, S.E. Tyaginov ${ }^{\mathbf{1} 2}$, B. Kaczer ${ }^{\mathbf{3}}$, M. Jech ${ }^{1}$, A. Chasin ${ }^{3}$, A. Grill ${ }^{1}$, G. Hellings ${ }^{3}$, M.I. Vexler ${ }^{2}$, D. Linten ${ }^{3}$, T. Grasser ${ }^{1}$

1 TU Vienna, Institute for Microelectronics, Vienna 1040, Austria

2 loffe Institute, 194021 St. Petersburg, Russia

${ }^{3}$ IMEC, Kapeldreef 75, 3001 Leuven, Belgium

Abstract For the first time, comprehensive modeling of hot-carrier degradation in FinFETs is performed. To achieve this goal, our physics-based hot-carrier degradation model, which considers multiple- and single-carrier mechanisms of $\mathrm{Si}-\mathrm{H}$ bond dissociation and all their superpositions, is used. The rates of these bond-breakage processes have been calculated by using the carrier energy distribution function which is obtained by solving the Boltzmann transport equation. The performed analysis of hot-carrier degradation in these transistors shows that the damage is localized in the upper part of the drain area of the fin. A very good agreement is also achieved between experimental changes of the drain current during stress and simulation results. It is important to emphasize that the model employs the same parameter set as that used to represent hot-carrier degradation in planar short-channel transistors and in high-voltage devices. 